\title{
Galaxy Threshing and the Origin of Intracluster Stellar Objects
}

\author{
Kenji Bekki, Warrick J. Couch \\ School of physics, University of New Southwales, Sydney, NSW, 2052, \\ Australia \\ Michael J. Drinkwater \\ Department of Physics, University of Queensland, Queensland 4072, \\ Australia \\ Yasuhiro Shioya \\ Astronomical Institute, Tohoku University, Sendai, 980-8578, Japan
}

\begin{abstract}
.
We numerically investigate the dynamical evolution of non-nucleated dwarf elliptical/spiral galaxies $(\mathrm{dE})$ and nucleated ones $(\mathrm{dE}, \mathrm{Ns})$ in clusters of galaxies in order to understand the origin of intracluster stellar objects, such as intracluster stars (ICSs), GCs (ICGCs), and "ultracompact dwarfs" (UCDs) recently discovered by all-object spectroscopic survey centred on the Fornax cluster of galaxies. We find that the outer stellar components of a nucleated dwarf are removed by the strong tidal field of the cluster, whereas the nucleus manages to survive as a result of its initially compact nature. The developed naked nucleus is found to have physical properties (e.g., size and mass) similar to those observed for UCDs. We also find that the UCD formation process does depend on the radial density profile of the dark halo in the sense that UCDs are less likely to be formed from dwarfs embedded in dark matter halos with central 'cuspy' density profiles. Our simulations also suggest that very massive and compact stellar systems can be rapidly and efficiently formed in the central regions of dwarfs through the merging of smaller GCs. GCs initially in the outer part of $\mathrm{dE}$ and $\mathrm{dE}$, Ns are found to be stripped to form ICGCs.
\end{abstract}

\section{UCD as an intracluster stellar object}

A new type of sub-luminous and extremely compact "dwarf galaxy" has recently been discovered in an "all-object" spectroscopic survey centred on the Fornax cluster of galaxies (Drinkwater et al. 2000). While objects with this type of morphology have been observed before - the bright compact objects discovered by Hilker et al. 1999 - and the very luminous globular clusters around cD galaxies (Harris, Pritchet, \& McClure 1995) - in this particular case they have been found to be members of the Fornax cluster, have intrinsic sizes of only $\sim 100 \mathrm{pc}$, 
and have absolute $B$-band magnitudes ranging from -13 to -11 mag. Hence Drinkwater et al. have named them "ultra-compact dwarf" (UCD) galaxies. Importantly, the luminosities of UCDs are intermediate between those of globular clusters and small dwarf galaxies and are similar to those of the bright end of the luminosity function of the nuclei of nucleated dwarf ellipticals.

The radial distribution, orbital velocity dispersion, and metallicity distribution of UCDs are suggested to provide valuable information on the difference in formation histories between UCDs, ICGCs, and ICSs (Bekki et al. 2003a). The "galaxy threshing" scenario (Bekki et al. 2001) has predicted that only luminous $\mathrm{dE}, \mathrm{Ns}$ with highly eccentric orbits and small pericenter distance from the center of a cluster can become UCDs after the outer dwarf envelopes are completed stripped by the cluster tidal field. ICGCs and ICSs have been demonstrated to form via tidal stripping of GCs and stars from cluster member galaxies (Bekki et al. 2003b). Here we reinvestigate the formation of UCDs/ICGCs/ICSs by using numerical simulations with larger number of particles (up to $N \sim 10^{6}$ ) to understand (1) how the formation histories of UCDs depend on the structure of dE,Ns (in particular, the central density of their dark matter halos, i.e., cores vs cusp), (2) the dynamical evolution of GCs in dEs orbiting the Fornax cluster , (3) whether these GCs can become UCDs in the center of dEs via merging of GCs. The details of the models for the Fornax cluster are given in Bekki et al. (2003a) and thus we briefly summarize the results here.

\section{Galaxy threshing and UCD/ICS formation}

Figures 1 and 2 summarize the dynamical evolution of the $\mathrm{dE}, \mathrm{N}$ model with $M_{\mathrm{B}}$ $=-16 \mathrm{mag}$, the NFW dark matter halo, and the nuclear mass fraction of 0.05 (referred to as the fiducial model, FO1). As the dE,N approaches the pericenter of its orbit, the strong global tidal field of the Fornax cluster stretches the envelope of the dE' $\mathrm{N}$ along the direction of the dwarf's orbit and consequently tidally strips the stars of the envelope $(T=1.13 \mathrm{Gyr})$. The dark matter halo, which is more widely distributed than the envelope due to its larger core radius, is also efficiently removed from the $\mathrm{dE}, \mathrm{N}$ during the pericenter passage. Since the envelope (and the dark matter halo) loses a significant fraction of its mass during the passage of the pericenter, the envelope becomes more susceptible to the tidal effects of the Fornax cluster after the pericenter passage. Therefore, each subsequent time the dwarf approaches the pericenter, it loses an increasingly larger fraction of its stellar envelope through tidal stripping (compare, for example, the $T=2.26$ and $T=2.83 \mathrm{Gyr}$ time points). Consequently, both the envelope and the dark matter halo become smaller, less massive, and more diffuse after five passages of the pericenter $(T=3.34 \mathrm{Gyr})$.

The stripped stars form a long tidal stream of ICSs along the "rosette" orbit within the orbital plane $(T=1.13 \mathrm{Gyr})$ and the ICSs can have metallicity of $[\mathrm{Fe} / \mathrm{H}] \sim-0.76$ for $B-V=1$ for the $\mathrm{dE}, \mathrm{N}$. The central nucleus, on the other hand, is just weakly influenced by the tidal force as a result of its compact configuration. Because of its strongly self-gravitating nature, the nucleus loses only a small amount $(\sim 20 \%)$ of its mass and thus maintains its compact morphology during its tidal interaction with the Fornax cluster. As a result, a very compact stellar system with a negligible amount of dark matter is formed 

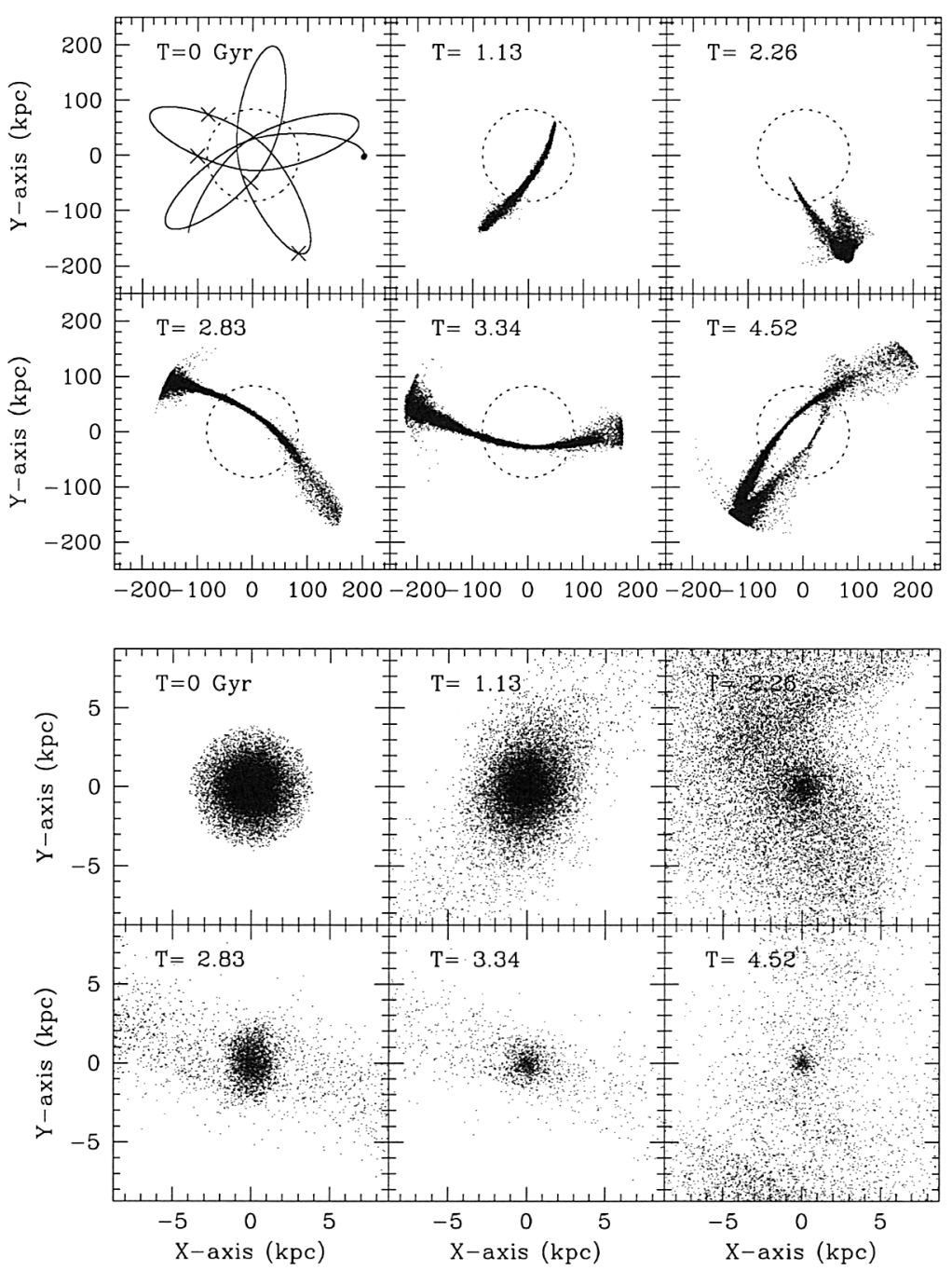

Figure 1. Morphological evolution of the stellar components (the stellar envelope and the nucleus) of the $\mathrm{dE}, \mathrm{N}$ projected onto the $x$ $y$ plane for the fiducial model (FO1). The time $T$ (in units of Gyr) indicated in the upper left corner of each frame represents the time that has elapsed since the simulation starts. Each frame is $500 \mathrm{kpc}$ on a side in the upper six panels and $17.5 \mathrm{kpc}$ on a side in the lower panels. The orbital evolution of the $\mathrm{dE}, \mathrm{N}$ at $4.5 \mathrm{Gyr}$ is indicated by the solid line in the upper left panel in the upper six panels. The location of the nucleus of the dwarf is indicated by crosses for $T=1.13,2.26$, 2.83, and 3.34 Gyr. The scale radius of the adopted NFW model for the dark matter halo distribution of the Fornax cluster mass profile is indicated by a dotted circle in each of the upper six panels. 


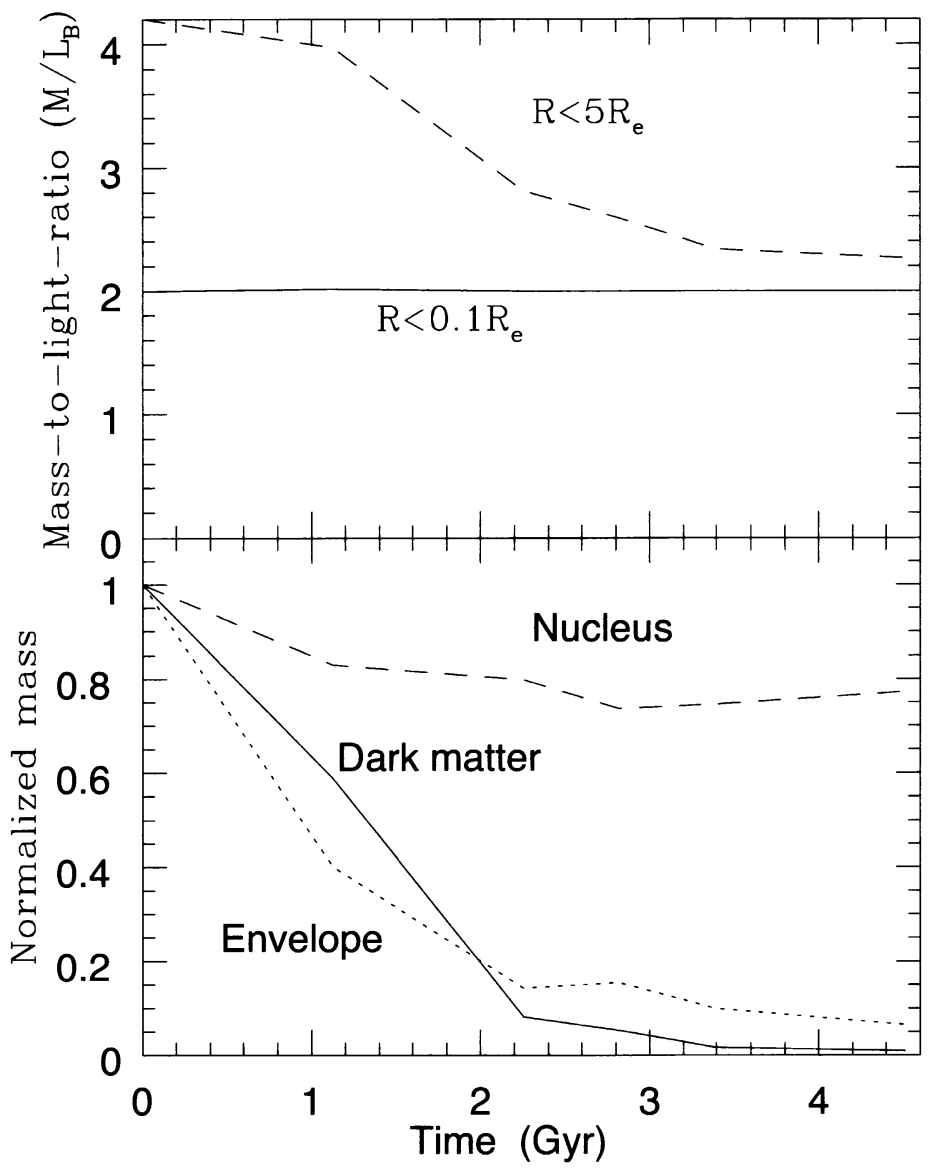

Figure 2. Time evolution of the $B$-band mass-to-light-ratio $\left(M / L_{\mathrm{B}}\right)$ (upper panel) and that of the total mass normalized to the initial mass for each collisionless component (lower panel) in the fiducial model (FO1). In the upper panel, $M / L_{\mathrm{B}}$ estimated for $R<0.1 R_{\mathrm{e}}$ (where $R$ and $R_{\mathrm{e}}$ are the distance from the center of the $\mathrm{dE}, \mathrm{N}$ and the initial effective radius of the $\mathrm{dE}, \mathrm{N}$, respectively) and for $5 R_{\mathrm{e}}$, are shown by the solid and dotted lines, respectively. In the lower panel, the total mass within $R<5 R_{\mathrm{e}}, R<R_{\mathrm{e}}$, and $R<0.1 R_{\mathrm{e}}$ for the dark matter halo, the stellar envelope, and the nucleus is shown by the solid, dotted and dashed lines, respectively. The mass-to-light ratio, $M / L_{\mathrm{B}}$, decreases dramatically from 4.2 to 2.3 for $r<5 r_{\mathrm{e}}$. This result clearly explains why the UCDs are observed to have mass-to-light ratios that are much smaller $\left(M / L_{\mathrm{B}}=2-4\right.$; D03) than those observed for dE,Ns $(\sim 10)$ for some of the Local group dwarf: galaxy threshing is the most efficient in the outer regions of a $\mathrm{dE}, \mathrm{N}$ where the dark matter halo dominates gravitationally. About $95 \%$ of the envelope initially within $r_{\mathrm{e}}$, and $20 \%$ of the nucleus initially within $0.1 r_{\mathrm{e}}$ are removed from the $\mathrm{dE}, \mathrm{N}$. 


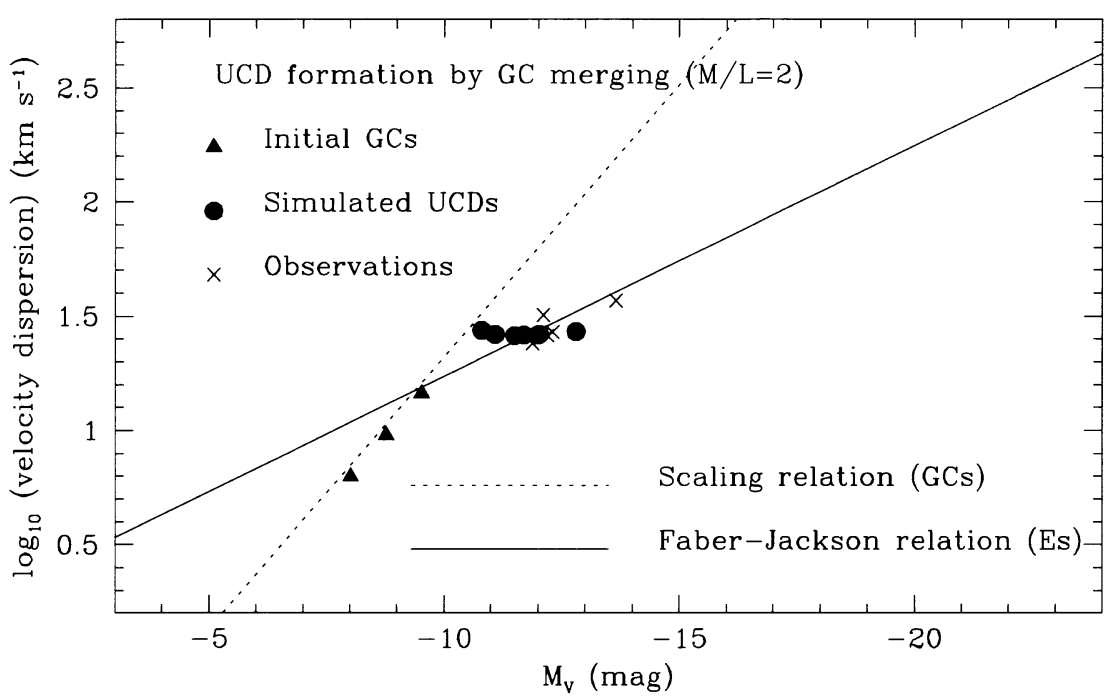

Figure 3. Distribution of UCDs formed from GC merging in dEs/dIs on the [central velocity dispersion, $M_{\mathrm{V}}$ ]-plane. Six UCDs formed in the central regions of dEs via GC merging are represented by the filled circles. The central velocity dispersion is given in units of $\mathrm{km} \mathrm{s}^{-1}$ and plotted on a $\log _{10}$ scale. For comparison, the observed UCDs (crosses) and the original GCs (filled triangles) are also plotted. The solid and the dotted lines represent the scaling relations for GCs (Djorgovski 1993) and elliptical galaxies (Faber \& Jackson 1976), respectively. The model and the method for deriving $M_{\mathrm{V}}$ and the central velocity dispersion of the UCDs formed from GC merging are given in Bekki et al. (2003a). The total number of GCs $\left(N_{\mathrm{GC}}\right)$ is 19 for the $\mathrm{dE}$ model with $M_{\mathrm{B}}=-16$ mag and $S_{\mathrm{N}}=5$ and these $N_{\mathrm{GC}}$ and $S_{\mathrm{N}}$ are considered to be free parameters in this numerical study. The initial GC mass ranges from $10^{5} M_{\odot}$ to $2 \times 10^{6} M_{\odot}$, and $N_{\mathrm{GC}}$ and $S_{\mathrm{N}}$ are changed according to the adopted mass of each GC. Note that the developed massive star clusters via GC merging, which can be identified as "nuclei" or "UCDs" in the center of dEs, have a scaling relation different from those of Es and GCs. This result implies that the observed location of UCDs in this plane can be understood in term of GC merging in the dE's central regions. The shapes of galactic nuclei (UCDs) depend on whether the host dwarfs have elliptical shapes (dE) or disky ones (dI). Some nuclei/UCDs have rotation and flattened shapes, in particular, for dIs with smaller number of GCs. The more detailed discussion on this result and on the relation of nuclei/UCDs to $\omega$ Cen and G1 are given in Bekki et al. (2003a). 
from the dE, $\mathrm{N}$ by $T=4.5 \mathrm{Gyr}$. The total mass and size of the remnant are $\sim 3.8 \times 10^{7} M_{\odot}$ and $\sim 100 \mathrm{pc}$ (five times the core or scale radius), consistent with the observed properties of a UCD. Hence this new study, based on fully self-consistent numerical models of $\mathrm{dE}, \mathrm{Ns}$, confirms the earlier results of Bekki et al. (2001) based on a more simplistic model.

\section{GC evolution in cluster dEs: UCD or ICGC formation ?}

Our simulations on GC merging in dEs/dIs have found the following interesting results: (1) Galactic nuclei or UCDs formed by 10-20 GC merging form a scaling relation that is different both from GCs and Es (Figure 3), (2) The shapes of the developed nuclei or UCDs depend on the host dwarf's luminosity in the sense that brighter dEs have more spherical nuclei/UCDs, (3) The brighter dEs are more likely to be transformed into dE,Ns, (4) The GCs in the outer part of dEs $\left(R>0.5 R_{\mathrm{e}}\right)$ are likely to be stripped to form ICGCs within a few Gyr (thus only central GCs can be merged into the nuclei/UCDs), and (5) Structure and kinematical properties of these ICGC systems depend on the orbital properties of their host dwarfs.

The present set of numerical simulations suggest that morphological evolution of dEs/dIs with GCs can be different after they enter a cluster environment: Some of them can be transformed into dE,Ns via GC merging in their central regions, if initial distributions of their GCs are more centrally concentrated. Such newly formed $\mathrm{dE}, \mathrm{Ns}$ can be furthermore transformed into UCDs due to the strong cluster tidal field, if it has lower dark matter density, highly eccentric orbits, and small pericenter distance. If $\mathrm{dE} / \mathrm{dIs}$ with GCs have more diffusely distributed GC systems, their GCs are tidally stripped to form ICGCs with the host dwarfs' $S_{\mathrm{N}}$ decreasing significantly. Some fraction of the observed ICSs can originate from the disrupted $\mathrm{dEs}$ and $\mathrm{dE}, \mathrm{Ns}$ (by galaxy threshing) in a cluster so that the spatial distribution, kinematics, and metallicity distribution of these ICSs reflect the orbital evolution and the disruption process of these dwarfs in the cluster. Thus we need to understand first the dynamical evolution of low luminosity cluster dwarfs, which are numerous and more susceptible to disruption by cluster global tidal field (and thus one of major sources of intracluster stellar objects) in order to understand the origin of UCDs, ICGCs, and ICSs.

\section{References}

Bekki, K., Couch, W. J., \& Drinkwater, M. J., 2001, ApJL, 552, 105

Bekki, K., Couch, W. J., Drinkwater, M. J., \& Shioya, Y., 2003a, MNRAS 344, 399

Bekki, K., Forbes, D. A., Beasley, M. A., \& Couch, W. J., 2003b, MNRAS 344, 1334

Drinkwater, M. J., Jones, J. B., Gregg, M. D., \& Phillipps, S., 2000b, PASA, 17,227

Harris, W. E., Pritchet, C. J., \& McClure, R. D., 1995, ApJ, 441, 120

Hilker, M., Infante, L., \& Richtler, T., 1999, A\&AS, 138, 55 